\title{
Article
}

\section{Treatment Outcomes of Anti-Neutrophil Cytoplasmic Autoantibody-Associated Vasculitis in Patients Over Age 75 Years: A Meta-Analysis}

Morris, A.D., Elsayed, M.E., Ponnusamy, A., Rowbottom, A., Martin, Francis L, Geetha, D. and Dhaygude, A.P.

Available at http://clok.uclan.ac.uk/32595/

Morris, A.D., Elsayed, M.E., Ponnusamy, A., Rowbottom, A., Martin, Francis L ORCID: 0000-0001-8562-4944, Geetha, D. and Dhaygude, A.P. (2020)

Treatment Outcomes of Anti-Neutrophil Cytoplasmic Autoantibody-Associated Vasculitis in Patients Over Age 75 Years: A Meta-Analysis. American Journal of Nephrology . ISSN 0250-8095

It is advisable to refer to the publisher's version if you intend to cite from the work. http://dx.doi.org/10.1159/000506532

For more information about UCLan's research in this area go to http://www.uclan.ac.uk/researchgroups/ and search for < name of research Group>.

For information about Research generally at UCLan please go to http://www.uclan.ac.uk/research/

All outputs in CLoK are protected by Intellectual Property Rights law, including Copyright law. Copyright, IPR and Moral Rights for the works on this site are retained by the individual authors and/or other copyright owners. Terms and conditions for use of this material are defined in the policies page. 


\section{Treatment outcomes of ANCA-associated vasculitis in patients over age 75 years: a meta-analysis}

Adam Morris ${ }^{1 *}$, Mohamed Elsayed ${ }^{1}$, Arvind Ponnusamy ${ }^{1}$, Anthony Rowbottom², Francis Martin ${ }^{3}$ Duvuru Geetha $^{4}$ \& Ajay Dhaygude ${ }^{1}$.

${ }^{1}$ Renal Medicine, Royal Preston Hospital, Preston, Lancashire, UK

${ }^{2}$ Department of Immunology, Royal Preston Hospital, Preston, UK

${ }^{3}$ School of Pharmacy \& Biomedical Sciences, University of Central Lancashire, UK

${ }^{4}$ Department of Medicine, Johns Hopkins University, Baltimore, USA

Short title: Treatment outcomes of AAV in patients $\geq 75$ years

\section{*Corresponding author:}

Dr Adam Morris

Department of Nephrology

Royal Preston Hospital, Lancashire NHS Foundation Trust

Sharoe Green Lane, Fulwood, Preston, PR2 9HT

E-mail: Adam.Morris@Ithtr.nhs.uk

Tel: 01772523748

Keywords: ANCA, Elderly, Immunosuppression, Outcomes, Vasculitis

Word Count: 4999 
1 Background: The benefits of treating ANCA-associated vasculitis (AAV) in advancing age remains unclear with 2 most published studies defining elderly as $\geq 65$ years. This study aims to determine outcomes of induction 3 immunosuppression in patients aged $\geq 75$ years.

4 Method: A cohort of patients aged $\geq 75$ years with a diagnosis of AAV between 2006-2018 was constructed from 5 two centres. Follow up was to two years or death. Analysis included multivariable Cox regression to compare 6 mortality and ESRD based on receipt of induction immunosuppression therapy with either cyclophosphamide or 7 rituximab. A systematic review of outcome studies was subsequently undertaken amongst this patient group 8 through Pubmed, Cochrane and Embase databases from inception until 16/10/19.

9 Results: 67 patients were identified. Mean age was $79 \pm 2.9$ years and $82 \%$ ( $n=55)$ received induction 10 immunosuppression. Following systematic review, four studies were eligible for inclusion, yielding a combined 11 total of 290 patients inclusive of our cohort. The aggregated one year mortality irrespective of treatment was $31 \%$ 12 (Cl 25\% - 36\%). Within our cohort, induction immunosuppression therapy was associated with a significantly lower 13 two-year mortality risk [HR $0.29(95 \% \mathrm{Cl} 0.09-0.93)]$. The pooled HR by meta-analysis confirmed this with a 14 significant risk reduction for death [HR 0.31 ( $\left.95 \% \mathrm{Cl} 0.16-0.57), I^{2}=0 \%\right]$. Treated patients had a lower pooled rate 15 of ESRD, but was not statistically significant [HR $0.71(95 \% \mathrm{Cl} 0.15-3.35)$ ].

16 Conclusion: This meta-analysis suggests that patients $\geq 75$ years with AAV do benefit from induction 17 immunosuppression with a significant survival benefit. Age alone should not be a limiting factor when 18 considering treatment. 


\section{Introduction}

ANCA-associated vasculitis (AAV) tends to present with rapidly progressive renal disease, carrying a significant risk of morbidity and mortality with a poorer survival probability in those patients requiring renal replacement therapy at presentation (1). Current established immunosuppressive therapies are effective with improved patient and renal survival $(2,3)$, but their use requires careful patient selection when balanced against the potential risks, with up to $60 \%$ of deaths in the first year resulting from adverse effects of treatment $(3,4)$. considering treatment in patients with $\operatorname{AAV}(5,6)$. This is based on the outcomes of previously published observational studies and randomised control trials, most of which tended to categorise older age as greater than 65 years and may not provide an accurate representation of those with advancing age $(4,6-8)$. Subsequently, despite being a disease that predominantly effects the elderly, as well as the most common cause of biopsy proven acute kidney injury in patients over the age of 80 years, the benefit of treating AAV in older age groups remains unclear (9-12). This study attempts to address this by evaluating treatment outcomes in patients $\geq 75$ years with AAV from two centres with subsequent systematic review and meta-analysis of the published literature.

\section{Materials \& Method}

\section{Participants \& Study design}

36 A cohort of consecutive patients aged $\geq 75$ years with a diagnosis of AAV between 2006-2018 was constructed

37 from two centres; one in the United Kingdom (UK) and one in the United States of America. All participants had renal impairment secondary to AAV at the time of diagnosis. Those with missing data or dual positivity for both ANCA and anti-GBM antibodies were excluded. For the remaining patients, the following data was retrospectively collected from the time of diagnosis; demographics, clinical presentation, modified Charlson comorbidity index (CCI) $(13,14)$, histopathology, immunosuppression therapy, patient outcomes and laboratory values including ANCA specificity, serum creatinine and estimated glomerular filtration rate (eGFR). As renal and connective tissue disease were the conditions of interest in our cohort, a modified $\mathrm{CCl}$ that did not include these in its calculation was used. Total score could range from 0 to 32. eGFR was calculated using the Modified Diet in Renal Disease equation (15). Cause of death was attained from review of medical records and categorised as follows; infection, active vasculitis, cardiovascular disease, respiratory disease, peripheral vascular disease, cerebrovascular disease, malignancy and unknown cause.

Patients were categorised into two groups; those who received induction therapy and those who did not. Induction therapy was defined as regimes utilising either cyclophosphamide or rituximab. The dosing regimen of intravenous cyclophosphamide adopted in both centres adjusted for renal function and patient age in accordance 
rituximab was administered at a dose of $375 \mathrm{mg}$ per square meter of body surface area per week for four weeks

53 or as $1 \mathrm{~g}$ every two weeks for two doses. Pulsed intravenous methylprednisolone and plasma exchange were

54 administered according to local physician discretion. The cumulative dose range of methylprednisolone was 0.5-

55 3g. Considerations for plasma exchange included dialysis dependence, serum creatinine $>500 \mu \mathrm{mol} / \mathrm{L}$ or

56 pulmonary haemorrhage. This retrospective cohort study received ethical approval from the UK Health Research

57 Authority and Confidentiality Advisory Group and institutional review board at Johns Hopkins Hospital.

\section{Outcomes}

The primary outcomes were risk of ESRD, death and the composite outcome of death or ESRD within two years of follow up. ESRD was defined by continued use of renal replacement therapy at follow up. Secondary outcomes included serious adverse events and renal recovery. Serious adverse events of therapy were defined as infection requiring hospitalisation, new onset malignancy, thrombocytopenia, leukopenia, bone marrow suppression and complications of glucocorticoid therapy including new onset diabetes mellitus, osteoporosis and osteoporotic fractures. Renal recovery was defined as sufficient improvement in renal function to achieve dialysis independence. Renal histopathology was categorised according to the Berden histopathological classification system (18).

\section{Systematic review \& study selection for meta-analysis}

68 A systematic review was undertaken to identify any studies evaluating outcomes in patients aged $\geq 75$ years with 69 AAV. Pubmed, Cochrane and Embase databases were each searched independently by two reviewers (AM \& ME) 70 from inception until 16.10.2019 using the following search strategy; "ANCA" OR "anti-neutrophil cytoplasmic antibody" OR "vasculitis" OR "PR3" OR "MPO" OR "ANCA-associated" AND "elderly" OR "old" OR "75 years" OR "geriatric" AND "ESRD" OR "end stage renal disease" OR "dialysis" OR "death" OR "survival" OR "mortality" OR "renal replacement therapy" OR "outcome". All outcome studies on patients $\geq 75$ years with AAV, inclusive of those presenting data as a subgroup analysis, were included. Case reports, editorials, letters to the editor, review articles, conference abstracts and studies not published in English were excluded from review. Eligible studies were independently screened and reviewed by two authors. In instances of disagreement, resolution by consensus was sought. The methodological quality and risk of bias of eligible studies was assessed using the Newcastle-Ottawa scale for observational studies. The protocol for this review was registered and published on PROSPERO (http://www.crd.york.ac.uk/PROSPERO/display record.php?ID=CRD42019123279).

Two investigators independently undertook data extraction using a predefined criterion. The study characteristics extracted for inclusion in meta-analysis were as follows; year of publication, study type, sample size of participants aged $\geq 75$ years, male percentage, the number of participants who received induction immunosuppression, follow up period, the rate of death and ESRD at one and two years, the rate of serious adverse events and the hazard ratios for death and ESRD in treated and untreated participants. 
86 Patient characteristics were presented as mean \pm SD or median for continuous variables and proportions for 87 categorical variables. A comparison between treated and untreated groups were analysed utilising t-Tests, Mann88 Whitney, chi-squared or Fisher exact tests where appropriate. Patient survival times were calculated from the 89 point of diagnosis until death, two years, loss to follow up or end of study $(01 / 12 / 2018)$. Renal survival was 90 calculated similarly with the addition of censor for death. The risk of death, ESRD and death or ESRD were studied using univariate and multivariable cox regression models, presented as hazard ratios (HR) with 95\% confidence intervals $(\mathrm{Cl})$. The following parameters were adjusted for in the final model; use of induction immunosuppression, gender, $\mathrm{CCl}$, eGFR at the time of diagnosis and the presence of renal limited disease. Gender and co-morbidity index were selected to reflect patient characteristics. eGFR was selected an indicator of disease severity and predictor of renal outcome. Renal limited disease was selected as a predictor variable as the absence of multi-system disease may confer a survival advantage. Univariate Kaplan-Meier curves were constructed to complement the cox regression hazard models for death, ESRD and death or ESRD. The rate of serious adverse events and impact of intravenous methylprednisolone were analysed utilising t-tests, Mann-Whitney, chi-squared or Fisher exact tests where appropriate.

The systematic review was conducted in accordance with PRISMA guidelines. A random effects meta-analysis model was used to calculate pooled HR for ESRD and death by treatment status. Study heterogeneity was evaluated using chi-square with a significance level of $P<0.10$ and $I^{2}$ statistics. Thresholds for $\mathrm{I}^{2}$ statistics were as follows; low (25-49\%), moderate (50-74\%) and high (>75\%).

\section{Results}

\section{Study Population}

106 Follow up data was completed in 67 patients aged $\geq 75$ years, of which $98.5 \%(n=66)$ had disease confirmed on renal biopsy. Descriptive baseline characteristics for this cohort according to treatment status are shown in Table 1. Mean age was $79 \pm 2.9$ years with a mean follow up period of $1.7 \pm 0.62$ years. Renal biopsy data was available in $94 \%$ of patients $(n=63$ ) with focal disease as the most common histological subtype as defined by the Berden classification (18). Just under half of patients had renal limited disease and ANCA serology was positive in $86.6 \%$ of patients ( $n=58$ ) with a predominance for MPO serotype.

112 Induction immunosuppression with cyclophosphamide or Rituximab was given to the majority of patients (82\%).

113 All received concomitant oral steroids and cyclophosphamide was the most commonly used agent with a median 114 cumulative dose of $2.73 \mathrm{~g}$ (interquartile range 7.14-1). 3 patients failed treatment with cyclophosphamide and 115 warranted continued therapy with rituximab. The non-induction cohort consisted solely of patients from the UK 116 centre. Amongst this group, three patients received alternative oral immunosuppression at the time of diagnosis; 117 two with azathioprine and steroids, one with steroids alone. From the induction and non-induction cohorts, 44 
118 and 9 patients were alive and dialysis independent at 6 months, respectively. Maintenance therapy amongst these

119 patients is shown in Table 1.

121 A total of thirty-three patients received intravenous methylprednisolone; $56.4 \%(n=31)$ vs. $16.7 \%(n=2)$ in the 122 induction and non-induction therapy cohorts respectively. Dosing data was available in twenty-nine patients with 123 mean dose of $2.24 \pm 0.8$ grams across both groups. Twelve (21.8\%) patients in the induction therapy cohort

124 received plasma exchange with treatment data available in ten cases. The median number of sessions 125 administered was five. No patients in the non-induction therapy cohort received plasma exchange. Of the 67 126 patients in our cohort, 26 were aged $\geq 80$ years with $88.5 \%(n=23)$ receiving induction immunosuppression.

\section{Outcomes of study population}

128 Clinical outcomes according to treatment status are outlined in Table 2. Three patients (4.5\%) died within the first

129 three months of diagnosis, of which only one received induction therapy. The overall one and two-year survival 130 rates irrespective of treatment were $79.1 \%(n=53)$ and $76.1 \%(n=51)$ respectively. The use of induction 131 immunosuppression was associated with a significant reduction in the risk of death [HR 0.29 (95\% Cl 0.09-0.93)]

132 (Table 3). Of the 16 deaths at the end of the two-year follow up period, one confirmed case was attributable to 133 underlying vasculitic disease. The leading cause of death in the non-induction cohort cannot be commented on 134 due to incomplete data with an unknown cause of death in $80 \%(n=4)$ of cases. Amongst those receiving induction 135 therapy, infection was the leading cause of death.

136 Eighteen patients (26.9\%) required dialysis within thirty days of their initial presentation, with no new cases of 137 dialysis dependence beyond this point throughout the follow up period. Of these patients, 15 received induction 138 immunosuppression, with four recovering renal function by twelve months. At the end of the two year follow up 139 period the rate of ESRD was $20 \%(n=11)$ and $16.7 \%(n=2)$ in the induction and non-induction cohorts respectively.

140 In multivariable cox regression analysis, renal survival was similar between the two groups [ $\mathrm{HR} 1.17$ (95\% Cl 0.25-

141 5.54)] (Table 3). A higher eGFR at the time of initial diagnosis was associated better renal survival [HR 0.75 (95\%

$142 \mathrm{Cl}$ 0.63-0.89)] (Table 3).

143

144 The therapeutic benefit of induction immunosuppression was maintained on assessing the composite outcome 145 of death or ESRD ([HR 0.33 (95\% Cl 0.12-0.86)] (Table 3). Supplementary figures 1-3 depicts univariate Kaplan-

146 Meier survival curves for death, ESRD and death or ESRD by induction immunosuppression.

147 On subgroup evaluation of patients $\geq 80$ years, $88 \% \quad(n=23)$ received induction immunosuppression with a 148 mortality rate of $21.7 \%(n=5)$ and all deaths occurring within 12 months of diagnosis. Acknowledging that analysis 149 may be limited the small sample size, use of induction immunosuppression did not confer a higher risk of death 150 in this group upon restricting the multivariable cox regression model to those aged $>80$ years $[\mathrm{HR} 0.01(95 \% \mathrm{Cl} 0-$ 151 0.84)] (supplementary Table1). No deaths or episodes of ESRD occurred within the first year in untreated patients $152 \geq 80$ years. 
153 No instances of death or ESRD occurred in those patients receiving rituximab. Meaningful analysis of

154 cyclophosphamide versus rituximab as outcome predictors was limited. Twenty-eight patients (41.8\%)

155 experienced serious adverse events, comprising of 22 patients from the induction cohort and 6 from the non-

156 induction cohort. Overall, infection accounted for the majority of cases. The use of induction immunosuppression

157 did not confer a higher risk of serious adverse events $(p=0.54)$. Similarly, the rate of adverse events did not

158 significantly differ between those patients who received intravenous methylprednisolone and those who did not;

$15942.9 \%(n=12)$ vs. $57.1 \%(n=16)$ respectively $(p=0.46)$.

\section{Systematic Review \& Study Selection}

161 The process of study selection is outlined in supplementary Figure 4. Thirteen citations qualified for full text

162 review. Nine were published as abstracts only and subsequently excluded. Characteristics of the four remaining

163 eligible studies are summarised in supplementary Table 2.

164 Only three studies categorised patients according to the use of immunosuppressive therapy; Bomback et al,

165 Weiner et al and Sato et al, with an aggregated total of 175 patients receiving induction immunosuppression (19-

166 21). The most commonly used agent was cyclophosphamide (72.6\%) with the majority receiving oral therapy

167 (59\%). Statistical analysis with stratification according to the use of induction immunosuppression was

168 undertaken by two studies; Weiner et al and Bomback et al $(19,20)$. The control group in Weiner et al consisted

169 of untreated patients as well as those receiving alternative regimes such as azathioprine, methotrexate or

170 mycophenolate. The control group in Bomback et al consisted of untreated patients only.

\section{Meta-analysis results}

172 With the addition of our cohort, a sample size of 290 patients aged $\geq 75$ years with AAV were available for review.

173 The one year mortality rate irrespective of treatment was 31\% (Cl 25\%-36\%). Weiner et al and Bomback et al both

174 used multivariable cox models to analyse the hazard of death and ESRD with induction therapy. Their results in

175 conjunction with the findings from our presented cohort were used to present the pooled HR for death and ESRD

176 by meta-analysis in 258 patients. The use of induction immunosuppression demonstrated a significant benefit for

177 patient survival with a pooled hazard ratio for death of 0.31 (95\% Cl 0.16-0.57) [ $I^{2}=0$ \%] (Figure 1). Induction

178 therapy was also associated with a lower pooled rate of ESRD, although not statistically significant [HR 0.71 (95\%

$179 \mathrm{Cl}$ 0.15-3.35)] (supplementary Figure 5). Serious adverse events were available for two studies with a combined 180 cohort of 105 treated patients and an incident rate of $38.1 \%(n=40)$.

\section{Discussion}

182 To date there has been limited published data guiding treatment outcomes of AAV in older populations and the

183 potential benefit of utilising established induction immunosuppression in those aged $\geq 75$ remains poorly defined. 
184 This represents an area of increasing clinical need owing to an overall rise in the both the incident and prevalence

185 of disease, with the former rising from 8-10/million to 13-20/million over recent years $(9,10,22)$. The present

186 study addresses this by reporting the experiences of two centres followed by a meta-analysis of published studies.

187 In doing so we identified a clear survival benefit in patients $\geq 75$ years treated with induction immunosuppression 188 with either Cyclophosphamide or Rituximab.

189 Within the first twelve months of treatment, the greatest risk to patient survival is adverse effects of therapy 190 rather than active vasculitis, with up to $60 \%$ of deaths resulting from infection $(4,8)$. It is considered that with 191 increasing age, a patient's ability to tolerate any significant immunosuppression is reduced with a higher 192 propensity to succumb to such adverse effects. Although not by study design, recent landmark trials in the 193 management of AAV have tended to only include patients under the age of 75 years $(17,23-25)$ and to date the 194 majority of observational studies reviewing the outcome of patients with AAV have often considered older age as 195 being greater than 65 years $(2,5-7,26,27)$. These have identified age $\geq 65$ years as a poor prognostic marker for 196 patient outcomes and in view of this, it would be anticipated that patients older than 75 years would fare even 197 worse.

198 In a retrospective single centre study evaluating one-year outcomes in patients over the age of 80 years, Bomback 199 et al demonstrated that treatment can prolong dialysis free survival with a remission rate of $49 \%$ and up to $37 \%$ 200 fewer patients reaching ESRD at one year compared to the untreated cohort (19). Although the cumulative 201 mortality rate was 49\%, with most patients dying from infection rather than underlying disease, the risk of death 202 at one year was $17 \%$ lower in treated patients (19). While this difference was not statistically significant, follow 203 up beyond one year identified a significantly lower risk for both ESRD and death. In a similar study, Weiner et al 204 evaluated the two-year survival in patients over the age of 75 years in a multi-centre retrospective study (20). 205 Their survival analysis supported that of Bomback et al, identifying superior patient survival in those given 206 induction therapy with 36\% fewer deaths and a significantly lower hazard ratio for death on multivariable analysis

207 (20). This remained unchanged on subgroup analysis of patients who received a lower cumulative dose of 208 cyclophosphamide.

209 Within our cohort analysis, we exhibited comparable findings to previous studies with a significantly lower hazard 210 ratio for death in treated patients. The survival benefit of induction immunosuppression persisted despite 211 advancing age following subgroup analysis of those aged $\geq 80$ years. There was no demonstrable benefit for renal 212 survival. The burden of co-morbidity was similar between the two groups and parallel to the findings of Bomback 213 et al, renal function at the time of diagnosis was predictive of outcomes. When considered in light of the findings 214 of Bomback et al, the current evidence suggests that on its own advancing age $\geq 80$ years should not discount 215 patients from treatment and that despite a higher potential risk of adverse effects, certain selected elderly 216 patients may benefit from induction immunosuppression in AAV.

217 The present meta-analysis of these observational studies confirmed a clear survival advantage of induction 218 immunosuppression over no/other oral immunosuppression in patients $\geq 75$ years with AAV. Similarly, a lower 
219 rate of ESRD with treatment was shown, although this did not reach statistical significance. In view of these

220 findings, the safe use a reduced dose of cyclophosphamide applied in both our cohort and Wiener et al suggests

221 that the dosing regimen described by previous studies can safely be adopted in older patients, with sufficient mitigation of risk without compromising therapeutic benefit $(4,6,7)$.

223 The remaining two studies of Hoganson et al and Sato et al identified on systematic review were not included in

224 meta-analysis $(21,28)$. Sato et al was a comparative study evaluating treatment outcomes of AAV in patients aged

$225 \geq 75$ years against those $<75$ years. In the cohort described, patients $\geq 75$ years numbered ten, of which $80 \%$ did

226 not receive induction therapy with either Cyclophosphamide or Rituximab (21). This was despite presenting with

227 more severe vasculitic disease due to the presumption that they were more susceptible to adverse events. The

228 implication of this selection bias is acknowledged by the authors when concluding a poorer survival rate in elderly

229 patients. The limitation of this study design could have affected our presented pooled one year survival rate,

230 however it did not have any implication on our subsequent meta-analysis with no stratification of outcomes by

231 treatment status. Similarly, data from Hoganson et al was excluded from meta-analysis on the same basis.

232 Despite improved patient survival with induction immunosuppression, the question remains, at what cost is this

233 achieved. Amongst other factors, advancing age has previously been associated with a higher degree of long term

234 damage and this patient group are potentially more frail with increased susceptibility to any potential treatment

235 related morbidity (29). In a follow-up study of their previously reported cohort, Weiner et al set out to address

236 this by evaluating the potential association between end organ damage and hospitalisation rates with therapy at

237 one and two years (30). In doing so, they identified that amongst patients $\geq 75$ years the use of cyclophosphamide

238 or rituximab was actually associated with a lower rate of damage (30). As disease severity at presentation is known

239 risk factor for permanent organ damage (29), this finding likely reflects the benefit of attenuated disease activity

240 achieved with therapy. There was no increased rate of hospitalisation or length of stay within 12 months of

241 treatment (30).

242 The potential for treatment related damage secondary to glucocorticoids is widely accepted and reported (29,

243 31). Although our study showed no increased risk of adverse event secondary to methylprednisolone exposure,

244 this is likely limited by our relatively small sample size. In their more recent study, Weiner et al did identify an

245 association between treatment related damage and fatal infections with a higher cumulative steroid exposure

246 within the first three months of therapy. Taking this into account in conjunction with the findings of our study, it

247 may be that future treatment strategies of AAV in advancing age would benefit more from minimising steroid

248 exposure, as opposed to avoidance or further modification of current therapy with rituximab or reduced dose

249 cyclophosphamide.

250 The findings of our study should be considered in context of its limitations. Firstly, the lack of randomised control 251 trials and the retrospective design of all included studies limits the level of evidence that could be derived from 252 them. Secondly, we acknowledge that the modified CCl scores observed in our cohort were seemingly low. In a 
253 previous small study applying a similar modified CCI to ours at diagnosis, with the exception of weighted score for

254 age, a higher score was associated with reduced patient and renal survival. In this study, patient age ranged from

255 18-76 years with a mean age of $53.2 \pm 15.63$ years and mean CCl at diagnosis of $4.9 \pm 2.49$ (14). The lower mean

$256 \mathrm{CCl}$ scores observed in our cohort would indicate a less comorbid and potentially less frail population, which would

257 favour better outcomes and should be taken into account when interpreting our cohort results. Thirdly, allocation

258 of treatment varied in each centre based on local expertise and clinical assessment which may have imposed a

259 significant selection bias: a factor evident by the imbalance of untreated patients between our two centres which

260 restricted adjustment for centre effect in the final models. This likely reflects individualisation of care based on

261 recognition of frailty and suitability of immunosuppressive therapy; an aspect of clinical assessment which is not

262 captured by measures such as the modified Charlson comorbidity index. A potential tool that could account for

263 this is the Clinical Frailty Scale, a frailty screening method that has recently been validated in patients with chronic

264 kidney disease. Its incorporation in future prospective studies could help stratify this crucial aspect of clinical

265 judgement, further guiding future immunosuppressive therapy in renal vasculitis (32). Fourthly, statistical analysis

266 to adjust treatment outcome according to the histological pattern of disease could not be undertaken due to

267 limited variability and small sample size. This is a factor which may have influenced treatment decisions. These

268 limitations should be weighed the rigorous and systematic approach of this study, as well as the previously limited

269 data guiding treatment in our defined population.

270 The question of whether or not induction immunosuppression is of more harm than benefit in patients with AAV

271 and advancing age is of increasing importance. The data presented here from our centres and pooled results from

272 meta-analysis suggests that patients $\geq 75$ years with AAV do benefit from induction immunosuppression, with a

273 significant survival advantage within the first two years of therapy. Age alone should not be a limiting factor when

274 considering treatment. Future trials in AAV may benefit from increasing the upper age limit at which patients are

275 considered elderly to 75 years in order to attain more representative data.

276 Acknowledgements: The authors would like to acknowledge the support of the renal department at Royal

277 Preston Hospital Lancashire NHS Foundation Trust in undertaking this study.

278 Ethics: This retrospective cohort study received ethical approval from the UK Health Research Authority and

279 Confidentiality Advisory Group and institutional review board at Johns Hopkins Hospital. The study was

280 conducted in accordance with the declaration of Helsinki.

281 Disclosures: Duvuru Geetha MD, MRCP (UK) is a Consultant ChemoCentryx. Ajay Dhaygude MD, MRCP (UK) has

282 received travel sponsorship from Pharmacosmos and lecture fees from MSD Pharma. The other authors have no

283 disclosures. All authors have no conflict of interest to declare. The results presented in this paper have not been published previously in whole or part, except in abstract format. 
285 Funding: This study received no external financial support.

286 Author contributions: Authors A.M, M.E, A.P and A.D were responsible for conception, design and oversight 287 of the study. A.M and D.G undertook data collection. A.M and M.E undertook systematic review, data analysis 288 and interpretation. A.M and M.E prepared the manuscript with critical review, contributions and approval from 289 all authors prior to final submission. 
291 1. de Joode AA, Sanders JS, Stegeman CA. Renal survival in proteinase 3 and myeloperoxidase ANCA-associated

292 systemic vasculitis. Clin J Am Soc Nephrol. 2013;8(10):1709-17.

293 2. Manno RL, Seo P, Geetha D. Older patients with ANCA-associated vasculitis and dialysis dependent renal 294 failure: a retrospective study. BMC Nephrol. 2015;16:88.

295 3. Lee T, Gasim A, Derebail VK, Chung Y, McGregor JG, Lionaki S, et al. Predictors of treatment outcomes in 296 ANCA-associated vasculitis with severe kidney failure. Clin J Am Soc Nephrol. 2014;9(5):905-13.

297 4. Little MA, Nightingale P, Verburgh CA, Hauser T, De Groot K, Savage C, et al. Early mortality in systemic 298 vasculitis: relative contribution of adverse events and active vasculitis. Ann Rheum Dis. 2010;69(6):1036-43.

299 5. Titeca-Beauport D, Francois A, Lobbedez T, Guerrot D, Launay D, Vrigneaud L, et al. Early predictors of one300 year mortality in patients over 65 presenting with ANCA-associated renal vasculitis: a retrospective, multicentre 301 study. BMC Nephrology. 2018;19(1).

302 6. Harper L, Savage CO. ANCA-associated renal vasculitis at the end of the twentieth century--a disease of older 303 patients. Rheumatology (Oxford). 2005;44(4):495-501.

304 7. Haris A, Polner K, Aranyi J, Braunitzer H, Kaszas I, Mucsi I. Clinical outcomes of ANCA-associated vasculitis in 305 elderly patients. Int Urol Nephrol. 2014;46(8):1595-600.

306 8. Flossmann O, Berden A, de Groot K, Hagen C, Harper L, Heijl C, et al. Long-term patient survival in ANCA307 associated vasculitis. Ann Rheum Dis. 2011;70(3):488-94.

308 9. Watts R, Lane S, Bentham G, Scott G. Epidaemiology of vasculitis a tend-year study in the united kingdom.

309 Arthritis Rheum. 2000;43(2):414-419

310 10. Watts RA, Al-Taiar A, Scott DG, Macgregor AJ. Prevalence and incidence of Wegener's granulomatosis in the 311 UK general practice research database. Arthritis Rheum. 2009;61(10):1412-6.

312 11. Mohammad AJ, Jacobsson LTH, Westman KWA, Sturfelt G, Segelmark M. Incidence and survival rates in 313 Wegener's granulomatosis, microscopic polyangiitis, Churg-Strauss syndrome and polyarteritis nodosa.

314 Rheumatology. 2009;48(12):1560-5.

315 12. Moutzouris DA, Herlitz L, Appel GB, Markowitz GS, Freudenthal B, Radhakrishnan J, et al. Renal biopsy in the 316 very elderly. Clin J Am Soc Nephrol. 2009;4(6):1073-82.

317 13. Charlson E, Pompei P, Ales K, MacKenzie R. A new method for classifying prognostic comorbidity in 318 longitudinal studies: development and validation. Journal of Chronic Disease. 1987;4095):373-383.

319 14. Ofer-Shiber S, Molad Y. Association of the Charlson comorbidity index with renal outcome and all-cause 320 mortality in antineutrophil cytoplasmatic antibody-associated vasculitis. Medicine (Baltimore).

321 2014;93(25):e152.

322 15. Levey A, Bosch J, Lewis J, Greene T, Rogers N, Roth D. A more accurate method to estimate glomerular 323 fitration rate from serum creatinine: a new prediction equation. Annals of Internal Medicine. 1999;130(6):461324470.

325 16. Mukhtyar C, Guillevin L, Cid MC, Dasgupta B, de Groot K, Gross W, et al. EULAR recommendations for the 326 management of primary small and medium vessel vasculitis. Annals of the Rheumatic Diseases. 2009;68(3):310327 7. 
17. Groot K, Harper L, Jayne D, Suarez L, Gregorini G, et al. Pulse versus daily oral cyclophsphamide for induction of remission in antineutrophil cytoplasmic antibody-associted vasculitis. Annals of Internal Medicine. 2009;150(10):671-680.

331 18. Berden AE, Ferrario F, Hagen EC, Jayne DR, Jennette JC, Joh K, et al. Histopathologic classification of ANCAassociated glomerulonephritis. J Am Soc Nephrol. 2010;21(10):1628-36.

333 19. Bomback AS, Appel GB, Radhakrishnan J, Shirazian S, Herlitz LC, Stokes B, et al. ANCA-associated 334 glomerulonephritis in the very elderly. Kidney Int. 2011;79(7):757-64.

335 20. Weiner M, Goh SM, Mohammad AJ, Hruskova Z, Tanna A, Bruchfeld A, et al. Outcome and treatment of 336 elderly patients with ANCA-associated vasculitis. Clin J Am Soc Nephrol. 2015;10(7):1128-35.

21. Sato S, Yashiro M, Matsuoka N, Asano T, Kobayashi H, Watanabe H, et al. Clinical features and outcomes in patients with elderly-onset anti-neutrophil cytoplasmic antibody-associated vasculitis. Geriatr Gerontol Int. 2018;18(10):1453-7.

340 22. Watts RA, Mahr A, Mohammad AJ, Gatenby P, Basu N, Flores-Suárez LF. Classification, epidemiology and 341 clinical subgrouping of antineutrophil cytoplasmic antibody (ANCA)-associated vasculitis. Nephrology Dialysis

342 Transplantation. 2015;30(suppl_1):i14-i22.

343 23. Stone J, Merkel P, Spiera, Seo P, Langford C, et al. Rituximab versus cyclophosphamide for ANCA-associated 344 vasculitis. N Eng J Med. 2010;363:221-232.

345 24. Jones R, Tervaert J, Hauser T, Luqmani R, Morgan M, et al. Rituximab versus cyclophosphamide in ANCA346 assocaited renal vasculitis. N Eng J Med. 2010;363:211-220.

347 25. Jayne DRW, Bruchfeld AN, Harper L, Schaier M, Venning MC, Hamilton P, et al. Randomized Trial of C5a 348 Receptor Inhibitor Avacopan in ANCA-Associated Vasculitis. J Am Soc Nephrol. 2017;28(9):2756-67.

349 26. Kaplan-Pavlovcic S. Clinical prognostic factors of renal outcome in anti-neutrophil cytoplasmic autoantibody 350 (ANCA)-associated glomerulonephritis in elderly patients. Nephrology Dialysis Transplantation.

$3512003 ; 18(90005): 5 v-7$.

352 27. Pagnoux C, Hogan SL, Chin H, Jennette JC, Falk RJ, Guillevin L, et al. Predictors of treatment resistance and relapse in antineutrophil cytoplasmic antibody-associated small-vessel vasculitis: comparison of two independent cohorts. Arthritis Rheum. 2008;58(9):2908-18.

355 28. Hoganson DD, From AM, Michet CJ. ANCA vasculitis in the elderly. J Clin Rheumatol. 2008;14(2):78-81.

356
29. Robson J, Doll H, Suppiah R, Flossmann O, Harper L, Hoglund P, et al. Glucocorticoid treatment and damage in the anti-neutrophil cytoplasm antibody-associated vasculitides: long-term data from the European Vasculitis Study Group trials. Rheumatology (Oxford). 2015;54(3):471-81.

30. Weiner M, Goh SM, Mohammad AJ, Hruskova Z, Tanna A, Sharp P, et al. Effect of Treatment on Damage and Hospitalization in Elderly Patients with Microscopic Polyangiitis and Granulomatosis with Polyangiitis. J Rheumatol. 2019.

31. Robson J, Doll H, Suppiah R, Flossmann O, Harper L, Hoglund P, et al. Damage in the anca-associated vasculitides: long-term data from the European vasculitis study group (EUVAS) therapeutic trials. Ann Rheum Dis. $2015 ; 74(1): 177-84$.

32. Nixon AC, Bampouras TM, Pendleton N, Mitra S, Dhaygude AP. Diagnostic Accuracy of Frailty Screening Methods in Advanced Chronic Kidney Disease. Nephron. 2019;141(3):147-55. 


\section{Figure Legends:}

Figure 1 - Forest plot of mortality risk in patients with ANCA-associated vasculitis aged $\geq 75$ years based on the use of induction immunosuppression

Supplementary Figure 1 - Kaplan-Meier survival curves for the outcome of death according to the use of induction immunosuppression therapy. Curves reflect univariate analysis

Supplementary Figure 2 - Kaplan-Meier survival curves for the outcome of end stage renal disease (ESRD) according to the use of induction immunosuppression therapy. Curves reflect univariate analysis

Supplementary Figure 3 - Kaplan-Meier survival curves for the composite outcome of death or end stage renal disease (ESRD) according to the use of induction immunosuppression therapy. Curves reflect univariate analysis

Supplementary Figure 4 - Flow diagram of systematic review and study selection for meta-analysis

Supplementary Figure 5 - Forest plot of ESRD risk in patients with ANCA-associated vasculitis $\geq 75$ years based on the use of induction immunosuppression 